\title{
A regulação em saúde: aspectos conceituais e operacionais
}

\author{
Health regulation: conceptual and operational aspects
}

Geisa Cristina Modesto Vilarins'; Helena Eri Shimizu², Maria Margarita Urdaneta Gutierrez³

' Mestre em Ciências da Saúde pela Universidade de Brasília (UnB) - Brasília (DF) Brasil.

ggvilarins@gmail.com

2 Pós-Doutora em Enfermagem pela Organização Pan-Americana de Saúde (OPAS) - Brasília (DF), Brasil. Professora Associada do Departamento de Saúde Coletiva, Faculdade de Ciências da Saúde da Universidade de Brasília (UnB) - Brasília (DF), Brasil.

shimizu@unb.br

${ }^{3}$ Doutora em Saúde Pública pela Escola Nacional de Saúde Pública da Fundação Oswaldo Cruz (ENSP/FIOCRUZ) - Rio de Janeiro (RJ), Brasil. Professora Adjunta do Departamento de Saúde Coletiva, Faculdade de Ciências da Saúde, Universidade de Brasília (UnB) - Brasília (DF), Brasil.

urdanetamm@gmail.com
RESUMO Entre os vários instrumentos de gestão utilizados no Sistema Único de Saúde (SUS), a regulação tem sido tratada com maior relevância. Este estudo objetivou uma revisão sobre o tema regulação em saúde e sua potencialidade para a execução das ações sanitárias. Foram selecionados 48 artigos, publicados em diversas bases de dados online, entre os anos 1989 e 2011. Por ser um mecanismo de equilíbrio entre oferta e demanda, a regulação busca a disponibilização de serviços e recursos assistenciais adequados às necessidades da população, garantindo um acesso de qualidade baseado nos princípios norteadores do SUS.

PALAVRAS CHAVE: Regulação em saúde; Acesso aos serviços de saúde; Gestão em saúde; Política pública de saúde.

ABSTRACT Among the various management tools used in the Unified Health System (SUS), regulation has been treated with greater relevance. This study aimed to review the issue on health regulation and its potential for implementation of health activities. Forty-eight articles were selected, published in several online databases, between 1989 and 2011. Because it is a balancing mechanism between supply and demand, regulation seeks the provision of healthcare services and resources tailored to the needs of the population, ensuring access to quality based on the guiding principles of SUS.

KEYWORDS: Health regulation; Health services accessibility; Health management; Health public policies. 


\section{Introdução}

A busca da equidade no acesso aos serviços de saúde é um objetivo explícito de muitos sistemas de saúde, no entanto, depara-se com muitas barreiras. A utilização do processo regulatório como instrumento de gestão pode se tornar um potente equalizador social do sistema de saúde para amortizar a desigualdade relacional entre os entes público e privado, além de atenuar a relação necessidade, demanda e oferta, tornando-a coerente, compatível e sem grandes distorçôes.

Cabe ressaltar que, no Brasil, a Constituição Federal de 1988 garante aos cidadãos o direito de acesso universal e integral aos cuidados de saúde, o que requer mudança significativa na forma de compreensão da regulação em saúde, visto que as atribuiçóes do Estado para garantir esse acesso ampliaramse significativamente.

Ademais, a descentralização das ações de saúde para estados e municípios determinou a divisão de responsabilidades e atribuiçôes entre os governos federal, estadual e municipal, assim como entre cidadãos, setor público e setor privado.

Apesar das controvérsias quanto à autoridade para regular, seja do Estado ou do município, permanece a convicção de que os provedores de serviços devem estar sob regulação, independentemente de serem entes públicos ou privados, uma vez que a ação regulatória otimiza os recursos disponíveis e favorece o devido acesso dos usuários.

$\mathrm{Na}$ área da saúde, o Estado é o principal sujeito da regulação, compreendendo três níveis de atuação: a) regulação sobre sistemas de saúde; b) regulação da atenção à saúde e; c) regulação do acesso à assistência ou regulação assistencial (SHILLING; REIS; MORAES, 2006). É importante ressaltar que a regulação sobre sistemas de saúde contém as ações de regulação da atenção à saúde, que, por sua vez, contêm as ações de regulação do acesso à assistência.

A regulação sobre sistemas de saúde tem como principais funções a definição de normas, monitoramento, fiscalização, controle e avaliação dos serviços de saúde. Essas funçôes são geralmente exercidas por diferentes órgáos reguladores, em âmbito nacional ou regional, incluindo o Ministério da Saúde e as agências reguladoras.

A regulação da atenção à saúde como ferramenta promotora de equidade, acessibilidade e de integralidade tem como objetivo a produção de açóes diretas e finais de atenção à saúde, e está direcionada aos prestadores de serviços de saúde públicos e privados. Os principais sujeitos são os gestores municipais e, de forma suplementar, os gestores estaduais e o gestor federal (SHILLING; REIS; MORAES, 2006). E suas principais funções são as ações de contratação, de controle, de regulação do acesso à assistência, de avaliação da atenção à saúde e de auditoria.

Por sua vez, a regulação assistencial promove a equidade do acesso aos serviços de saúde, garantindo a integralidade da assistência e permitindo ajustar a oferta assistencial disponível às necessidades imediatas do cidadáo, de forma equânime, ordenada, oportuna e racional. Para tanto, uma das estratégias utilizada pelo SUS é a organização de centrais de regulação do acesso por temas ou áreas assistenciais.

Apesar dos avanços na forma de operacionalização da regulação assistencial, o processo de regulação em saúde ocorre ainda de forma bastante incipiente, requerendo aprimoramento em diversos aspectos. É importante dotar esse processo de instrumentos que garantam, de acordo com a necessidade de cada caso, a qualidade do atendimento aos pacientes e a alocação eficiente dos recursos médico-hospitalares.

Justifica-se o interesse pelo tema proposto ao se notar o acesso dos usuários aos serviços do Sistema Único de Saúde (SUS) de forma não equânime, realidade percebida nos serviços públicos da saúde de todo o Brasil quando são noticiados os problemas decorrentes da gestão pública em saúde.

Além disso, associa-se a não aplicabilidade dos conceitos de regulação na prática vigente das políticas públicas em saúde, apesar de ser um poderoso instrumento de gestão.

Portanto, este estudo tem como objetivo apresentar uma breve revisão sobre o tema regulação, com enfoque em saúde, a fim de identificar as suas fragilidades e potencialidades. 


\section{MÉTODO}

Trata-se de uma pesquisa bibliográfica em que foram utilizadas as bases de dados online Web of Science, SciELO, Proquest, LILACS (Literatura Latino-Americana e do Caribe em Ciências de Saúde) e PubMed.

Realizou-se, inicialmente, uma análise da produção do conhecimento relacionada à regulação do acesso aos serviços públicos de saúde. Em seguida, foram identificadas as concepçóes sobre essa ferramenta de gestão referidas em periódicos de circulação nacional e internacional, além de em dissertaçóes de mestrado e teses de doutorado.

Os títulos e os resumos dos artigos foram considerados para a seleção ampla dos trabalhos, sendo destacados aqueles que apresentaram os seguintes descritores: regulação do acesso, gestão em saúde, sistemas de saúde e políticas públicas de saúde. Na sequência, as publicaçôes foram submetidas a releituras, com a finalidade de realizar uma análise interpretativa guiada pelos objetivos previamente estabelecidos, e agrupadas de acordo com os aspectos conceituais apresentados.

\section{Resultados e discussão}

Foram selecionados 48 artigos publicados entre os anos 1989 e 2011. Posteriormente, os mesmos foram agrupados de acordo com os seguintes eixos temáticos: a) aspectos conceituais da regulação; b) regulação em saúde.

Após submetidos à análise, foi possível identificar que 19 abordavam conceitos de regulação em diversas áreas do conhecimento, 23 aludiam especificamente à regulação em saúde e 06 , apesar de constarem nas bases de dados, não apresentavam o conteúdo pesquisado.

\section{Aspectos conceituais da regulação}

O conceito de regulaçáo vem, ao longo do tempo, sendo aprimorado e usado conforme as mudanças políticas, sociais e econômicas (BARBIERI; HORTALE, 2002).

De um modo geral, os estudos na área de regulação têm enfocado especialmente sua dimensão econômica.
Sob a ótica da teoria econômica clássica, a regulação poderia ser caracterizada como a intervenção estatal para corrigir 'falhas de mercado', utilizando instrumentos como incentivos financeiros e de comando e controle (SANTOS; MERHY, 2006). Tais falhas ocorrem quando as perfeitas condições de competição, voltadas para a 'lei da oferta e da demanda', náo estáo plenamente satisfeitas.

Destarte, a regulação é vista como um conjunto de açóes-meio que dirigem, ajustam, facilitam ou limitam determinados processos para o alcance de resultados que podem estar relacionados à satisfaçáo do consumidor ou ao atendimento das necessidades mais prementes de uma população (SHILLING; REIS; MORAES, 2006).

Ainda nessa linha de raciocínio, a regulação aparece como uma maneira de propiciar eficiência econômica e estaria a serviço dela (SALGADO, 2003), podendo ser entendida como um instrumento essencial para a manutenção do equilíbrio de qualquer sistema. Semelhantemente, Crozier (1989) enfatiza a regulação como um dos mecanismos corretores de que dispóe uma nação para controlar seus sistemas.

Desta forma, a regulação, por meio dos órgãos reguladores, pode identificar as perturbaçóes geradas pelo sistema, além de analisar e tratar as informaçóes relativas a um estado de desequilíbrio, e transmitir um conjunto de ordens coerentes a um ou vários dos seus órgãos executores (BARROSO, 2005). Ela promoveria, assim, o bem-estar de consumidores e usuários, ao mesmo tempo que incentivaria investimentos necessários para o desenvolvimento econômico.

Pressupôe-se, portanto, a existência de um sistema cujo funcionamento seja regulado a partir de regras e parâmetros acordados entre as partes executoras e reguladoras.

A regulação abarcaria, então, tanto o ato de regulamentar/elaborar leis, regras, ou normas, quanto as ações e técnicas que asseguram o cumprimento dessas leis, quais sejam fiscalização, controle, avaliação, auditoria, sanções e premiações (SHILLING; REIS; MORAES, 2006). Assim compreendido, o termo regulação é frequentemente usado quando se refere aos serviços que funcionam sob concessáo do Estado para suprir necessidades da população. 
Nesse contexto, o Estado pode transferir açóes para o setor privado ou agir em parceria com agentes sociais. Transforma-se, portanto, o antigo Estado centralizador da produção do bem público em um moderno Estado coprodutor, mas ainda na condição de responsável último por sua produção.

Logo, a regulação pode ser vista como a influência deliberada e propriamente dita do Estado em qualquer área ou setor que influencie a sociedade. $\mathrm{O}$ papel do Estado, ao se utilizar da regulação, é o de definir os critérios de organização e prestação dos serviços, estabelecendo prioridades, além de elaborar as regras para a atuação dos mercados.

A ação do Estado em função do interesse público é observada quando a regulação, basicamente, se referir à ação de uma agência pública sobre serviços de valor para a coletividade. Essa ação presume uma autoridade pública formalmente constituída que centraliza as açóes de regulação, atuando por fora das relaçóes comerciais e governamentais (WALSHE, 2003).

Indistintamente, no Brasil, segundo Ibanhes et al (2007), a regulação tem como fundamentação a macropolítica de ajuste econômico, de viés neoliberal, a partir da privatização de setores estratégicos como as áreas de energia, telecomunicações, petróleo e gás, vigilância sanitária e saúde suplementar.

Portanto, um Estado regulador apresenta como características primordiais a definição de grandes orientaçóes e alvos a serem alcançados no estabelecimento de políticas públicas, além de um sistema de monitorização e de avaliação para a análise dos resultados esperados.

\section{A regulação em saúde}

A garantia do acesso da população aos serviços públicos de saúde a uma assistência qualificada, por meio de uma rede organizada de serviços, requer a atuação direta do Estado na busca do estabelecimento de regras definidas para atuação dos mercados, o que configura a passagem de um Estado prestador para um Estado regulador, de fato.

Como efetivador das políticas públicas em saúde, o Estado utiliza a regulação do acesso aos serviços públicos como um poderoso instrumento de gestão. Todavia, há que se debater a utilização desse instrumento sob a ótica da transição do Estado provedor para o Estado garantidor da produção dos serviços públicos, visto que a regulação interfere na prestação desses serviços.

Sob essa lógica, a função reguladora do Estado é fundamental para harmonizar e articular a oferta e a demanda, pois a intervenção estatal na saúde seria a única forma de otimizar a alocação dos escassos recursos disponíveis para a sociedade (CASTRO, 2002).

Por conseguinte, a regulação, além de se referir aos macroprocessos de regulamentação, também define os mecanismos utilizados na formatação e no direcionamento da assistência à saúde propriamente dita (ANDREAZZI, 2003).

Registra-se que alguns países têm se preocupado mais enfaticamente com a questão da regulação em saúde. A Bulgária, por exemplo, compartilha com muitos outros países da Europa Central e da Oriental o desafio de passar de um sistema de saúde em que o Estado era o principal financiador e fornecedor de serviços de saúde para um sistema mais pluralista, com uma variedade de fontes de financiamento, incluindo um sistema de seguro de saúde e um papel mais forte do setor privado. Para tanto, a regulação do acesso foi utilizada como instrumento de gestão sobre os prestadores de serviços em saúde para se estabelecer um sistema de financiamento sustentável (RECHEL; BLACKBURN; SPENCER, 2011).

$\mathrm{Na}$ Inglaterra, a regulação, além de fornecer acesso aos serviços de saúde, é considerada também um instrumento na gestão de reclamaçôes sobre a saúde pública, disponibilizando dados para os serviços de ouvidoria e de assistência social (HOLMES-BONNEY, 2010). Outro ponto considerado no Serviço Nacional Britânico de Saúde (NHS) é que a regulação traz consigo o ônus de prestar as informaçóes para auditoria e fiscalização, além de respaldar a prestação de contas dos serviços de saúde.

No Brasil, o debate mais aprofundado com relação ao conceito, às práticas e às finalidades da regulação, do controle, da avaliação e da auditoria em saúde iniciou-se a partir de 2001 com as Normas Operacionais de Assistência à Saúde (NOAS). As NOAS SUS 
01/2001 ampliam a responsabilidade dos municípios sobre a atenção básica e reservam à Uniāo e aos Estados a gestão da média e da alta complexidade dos serviços públicos de saúde.

A regulação no SUS ganha força após o Pacto de Gestão (2006), que tem como principal finalidade a busca de maior autonomia para os estados e municípios no que tange aos processos normativos do SUS, definindo a responsabilidade sanitária de cada esfera de governo e tornando mais claras as atribuiçóes de cada uma, contribuindo, assim, para o fortalecimento da gestão compartilhada.

Portanto, permanece a convicção de que o Estado possui autoridade sobre os provedores de serviços - públicos e privados - para regular suas atividades, de modo a reduzir os custos de operação do sistema, garantindo aos cidadãos um atendimento gratuito de qualidade (ARRETCHE, 2003).

Ademais, o processo de regulação poderá interrelacionar a coordenação de atividades, a alocação de recursos e a administração de conflitos, além de utilizar diferentes técnicas, voltado para a busca de equilíbrio entre oferta, demanda e financiamento, com o objetivo de alcançar a eficiência e a equidade.

Apesar disso, a prestação de serviços na área da saúde apresenta-se como um dos setores mais problemáticos para o exercício da regulação pelo Estado. Regular não se resume ao ato de regulamentar, mas também inclui uma gama de açôes que verificam se a produção em saúde se dá conforme as regras estabelecidas (SHILLING; REIS; MORAES, 2006).

A regulação controla não só o cumprimento das leis para a proteção dos indivíduos e das comunidades carentes, como também controla a qualidade dos serviços. Claramente, a regulação tem um papel importante na proteção da população, impedindo fraudes e garantindo padrốes mínimos de qualidade dos serviços de saúde (SCRIVEN, 2007). Trata-se de um processo pelo qual a atividade do setor público e as forças de mercado são direcionadas para o bem público (JEWEL; WILKINSON, 2008).

Neste sentido, no final dos anos 1990, por meio da Lei no 9.782/99, criou-se a primeira agência reguladora social no Brasil: a Agência Nacional de Vigilância
Sanitária (ANVISA), com vistas a atuar não em um setor específico da economia, mas em todos os setores relacionados a produtos e serviços que podem afetar a saúde da população brasileira. Uma das singularidades dessa Agência é a sua competência tanto na regulação econômica do mercado (definição de preços e monitoramento do mercado) quanto na regulação sanitária (registros de medicamentos, por exemplo).

Outra agência reguladora criada foi a Agência Nacional de Saúde Suplementar (ANS), por meio da Lei no 9.961/00, com a finalidade de regulamentar os planos privados de saúde. Ela tem desenvolvido mecanismos tanto para as formulaçóes de políticas públicas de saúde quanto para a busca de melhor atendimento do beneficiário, com o intuito de procurar melhor balanceamento entre os procedimentos de mercado das operadoras e os direitos da sociedade de receber melhor qualidade na assistência à saúde (DIAS, 2004).

Com o entendimento do Ministério da Saúde (BRASIL, 2005, p. 318) e a anuência do Conselho Nacional de Secretários Municipais de Saúde (CONASEMS), a regulação é definida, então, como um conjunto de relaçôes, saberes, tecnologias e açôes que intermedeiam a demanda dos usuários por serviços de saúde e o acesso a estes.

Criada em 2008, a Política Nacional de Regulação do Ministério da Saúde (BRASIL, 2008) objetiva, precipuamente, promover o acesso equânime, universal e integral dos usuários ao SUS. Não em uma lógica meramente financeira, a regulação em saúde contribui para otimizar a utilização dos serviços, em busca da qualidade da ação, da resposta adequada aos problemas clínicos e da satisfação do usuário, sem que haja, para tanto, a fragmentação do cuidado.

No estado de Minas Gerais, a regulação é vista como um conjunto de regras impositivas de proteção com vistas ao monitoramento e ao controle dos serviços prestados. Nesse plano, o objetivo pode ser de caráter social ou econômico, e, em geral, visa a encorajar atividades consideradas úteis (MENICUCCI, 2005).

Em consonância, na região metropolitana de São Paulo, a regulação da saúde pública é apontada como possuidora de um caráter racionalizador dos serviços complementares e suplementares (FIANI, 2004). 
Como forma de operacionalizar o processo de regulação do acesso aos serviços de saúde, observa-se a criação de várias centrais em todo o país nas seguintes áreas: urgência e emergência, consultas e exames de média e alta complexidade, internações clínicas ou cirúrgicas e em terapia intensiva, transplantes, obstetrícia e neonatal, entre outras (EVANGELISTA; BARRETO; GUERRA, 2008).

Como exemplo, tem-se o Complexo Regulador do Distrito Federal, institucionalizado em 2009, apesar de as atividades de cunho regulatório terem sido iniciadas em 2006. Tal serviço é responsável por um conjunto de estratégias e açóes definidas em um plano de regulaçáo assistencial, para todos os níveis de complexidade, visando à organização efetiva de uma rede pública articulada hierarquicamente, a partir do planejamento estruturado em bases regionais. Tem a missão de fornecer o acesso devido aos usuários, por meio de centrais de regulação na área ambulatorial, nas especialidades de dermatologia, oftalmologia, radiologia e cardiologia, e na área de internação, com ênfase no acesso aos leitos de terapia intensiva.

Outro modelo é a central de regulação no estado do Ceará, sediada em Fortaleza, voltada para a regulação do acesso à terapia intensiva, com a disponibilização de leitos públicos e privados. Um estudo desenvolvido nesse cenário discute a relação entre o público e o privado na prestação dos serviços, além de abordar o papel do Estado e da sociedade civil na exigência de um controle efetivo sobre a utilização dos serviços oferecidos (CAVALCANTE; OLIVEIRA, 2011).

Sob o prisma da equidade na assistência à saúde, a regulação é imprescindível e sua finalidade é assegurar que se atinjam os grandes objetivos sociais do sistema de saúde, equilibrando as numerosas falhas de mercado e/ou falhas do governo que caracterizam esse setor (FARIAS, 2011).

Não obstante, o processo de regulação do acesso aos serviços de saúde pode constituir-se em um poderoso instrumento de intervenção na realidade sanitária, permitindo às instâncias de gestão estadual, municipal e federal regular o perfil assistencial mais adequado às necessidades de saúde.
Contudo, torna-se imperativo que o processo regulatório deva ser flexível para permitir sua adequação às peculiaridades municipais, respeitando as especificidades locais, ao mesmo tempo que subsidie decisóes sobre macropolíticas regionais e intersetoriais (CAVALCANTE, 2003).

Caracteriza-se, portanto, como um instrumento de gestão com potência para sinalizar, de forma sistematizada, os pontos de estrangulamento com vistas à consolidação do acesso às tecnologias de saúde existentes de forma mais equitativa e justa para a população.

\section{Considerações finais}

Nota-se a nítida polissemia do termo regulação, entretanto, é na área econômica que o mesmo encontra maior ressonância, como um instrumento de equilíbrio entre oferta e demanda, de modo a oferecer eficiência ao sistema com geração de resultados positivos.

$\mathrm{Na}$ área da saúde, a literatura demonstra que, sob o aspecto da oferta, a regulação busca a disponibilização de serviços e recursos assistenciais adequados às necessidades da população, com base em critérios epidemiológicos, mas que ainda encontra-se incipiente no SUS. Sob a ótica da demanda, a regulaçâo busca qualificá-la, disponibilizando o serviço de saúde mais adequado ao usuário, em momento oportuno, equânime, e pautado por critérios de priorização de riscos, o que também requer aprimoramentos.

Deve-se pensar na regulação em saúde sempre no contexto dos princípios norteadores do SUS, e não apenas como forma de racionalizar os recursos existentes. Sob essa lógica, o processo regulatório deverá estabelecer um redimensionamento da oferta (diminuição ou expansão), qualificando a utilização dos recursos assistenciais e financeiros e coibindo fluxos paralelos, baseados em relaçóes pessoais e outros critérios não científicos ou não pactuados.

Destarte, o papel da regulação revela-se imperativo ao promover a articulação e a integração das atividades de regulação com as açóes de fiscalização, 
controle, avaliação e auditoria nos diversos níveis de complexidade da assistência dentro de uma rede hierarquicamente organizada. E, ao garantir o acesso dos usuários aos serviços de saúde, atua sobre a oferta dos mesmos e estabelece a adequação dessa oferta às necessidades identificadas.
Promove, assim, a equidade do acesso e garante a integralidade da assistência de forma universal e ordenada, segundo os princípios do SUS. Torna-se, então, um instrumento de gestão pública imprescindível para garantir maior efetividade às açóes desenvolvidas pelos sistemas de saúde.

\section{Referências}

ANDREAZZI, M.F.S. Mercado de Saúde Suplementar: amplitudes e limites na arena da regulação. In: MONTONE, J.; WERNECK, A. (orgs.). Documentos técnicos de apoio ao Fórum de Saúde Suplementar de 2003. Rio de Janeiro: Ministério da Saúde, Agência Nacional de Saúde Suplementar, 2004. p. 121 146.

ARRETCHE, M. Financiamento federal e gestão local de políticas sociais: o difícil equilíbrio entre regulação, responsabilidade e autonomia. Ciência e saúde coletiva, Rio de Janeiro, v.8, n.2, p. 331-345, 2003.

BARBIERI, A.R.; HORTALE, V.A. Relações entre regulação e controle na reforma administrativa e suas implicações no sistema de saúde brasileiro. Revista de Administração Pública, Rio de Janeiro, v.36, n.2, p. 181-194, 2002.

BARROSO, J. O estado, a educação e a regulação das políticas públicas. Educação e Sociedade, Campinas, v.26, n.92, p. 725751, 2005.

BRASIL. Constituição da República Federativa do Brasil. Brasília: Saraiva, 2006

Ministério da Saúde. Conselho Nacional de Secretários Municipais de Saúde. O SUS de A a Z: garantindo saúde nos municípios. Brasília: Ministério da Saúde, 2005. 240p.

Ministério da Saúde. Portaria GM/MS n. 1.559, de 1.0 de agosto de 2008. Institui a Política Nacional de Regulação do Sistema Único de Saúde. Brasília: Diário Oficial da União, 4 ago. 2008.

CASTRO, J.D. Regulação em saúde: análise de conceitos fundamentais. Sociologias, Porto Alegre, v.4, n.7, p. 122-135, 2002.

CAVALCANTE, C.S.; OLIVEIRA, L.C. Regulação Assistencial: estratégia de publicização do acesso a leitos de Terapia Intensiva em Fortaleza, Ceará. Saúde em Debate, Rio de Janeiro, v.35, n.89, p.321-330, 2011.
CAVALCANTE, M.T.L. Cartão Nacional de Saúde e Central de Regulação de Ações de Saúde: Tendências nas Tecnologias de Informação em Saúde. 2003. 96f. Dissertação (Mestrado em Saúde Pública) - Fundação Oswaldo Cruz, Rio de Janeiro, 2003.

CROZIER, M. Estado modesto, Estado moderno: estratégia para uma outra mudança. Brasília: FUNCEP, 1989.

DIAS, R.D.M. A informação na regulação da saúde suplementar. 2004. 137f. Dissertação (Mestrado em Saúde Pública) - Fundação Oswaldo Cruz, Rio de Janeiro, 2004.

EVANGELISTA, P.A.; BARRETO, S.M.; GUeRRA, H.L. Central de Regulação de leitos do SUS em Belo Horizonte, Minas Gerais, Brasil: avaliação de seu papel pelo estudo das internações por doenças isquêmicas do coração. Caderno de Saúde Pública, Rio de Janeiro, v.24, n.4, p. 767-776, 2008.

FARIAS, S.F. et al. A regulação no setor público de saúde no Brasil: os (des) caminhos da assistência médico-hospitalar. Ciência e saúde coletiva, Rio de Janeiro, v. 16, supl. 1, s1043-1053, 2011.

FIANI, R. Afinal, a quais interesses serve a regulação? Economia e Sociedade, Campinas, v.13, n.1, p. 81-105, 2004.

HOLMES-BONNEY, K. Managing complaints in health and social care. Nursing Management, Harrow, London, v. 17, n. 1, p. 12-15, apr. 2010.

IBANHES, L.C. et al. Governança e regulação em saúde: desafios para a gestão na Região Metropolitana de São Paulo, Brasil. Caderno de Saúde Pública, Rio de Janeiro, v.23, n. 3, p. 575-584, 2007.

JEWEL, T;; WILKINSON, J. Health and social care regulation in Wales: an integrated system of political, corporate and professional governance for improving public health. The Journal of the Royal Society for the Promotion of Health, v.128, n.6, p.306-312, 2008.

MENICUCCI, T.M.G. Regulação da Assistência à Saúde: o caso de Minas Gerais. Belo Horizonte: Fundação João Pinheiro, 2005. 152p. 
RECHEL, B.; BLACKBURN, C.M.; SPENCER, N.J. Regulatory barriers to equity in a health system in transition: a qualitative study in Bulgaria. Biomedical Central Health Service Research, London, v.11, n. 219, p. 1-10, 2011.

SALGADO, L.H. Agências regulatórias na experiência brasileira: um panorama do atual desenho institucional. Brasília: Instituto de Pesquisa Econômica Aplicada, 2003. 52p.

SANTOS, F.P.; MERHY E. E. A regulação pública da saúde no Estado brasileiro: uma revisão. Interface, Botucatu, v.10, n.19, p.25-41, 2006.
SCHILLING, C.M.; REIS, A.T.; MORAES, J.C. (orgs.). A política regulação do Brasil. Brasília: OPAS, 2006. 116 p.

SCRIVEN, E. The future of regulation and governance. The Journal of the Royal Society for the Promotion of Health, v.127, n.2, p. 72-77, 2007.

WALSHE, K. Regulating healthcare: a prescription for improvement? Philadelphia: Open University Press, 2003. 224p.

Recebido para publicação em Janeiro/2012 Versão definitiva em Novembro/2012.

Suporte financeiro: não houve

Conflito de interesse: inexistente 